\title{
FINITE ELEMENT MODELING EXPERIMENTS OF UNSTEADY FLOW AROUND A CIRCULAR CYLINDER
}

\author{
D.C. Lobão ${ }^{1}$ \\ ${ }^{1}$ Department of Exact Science, Federal Fluminense University, Volta Redonda, RJ, \\ Brazil,(lobaodiomarcesar@yahoo.ca)
}

\begin{abstract}
The finite element method (FEM) has become one of the most important and useful engineering tools for engineers and scientists in the last three decades. Finite element method is considered very powerful and efficient tool in solving partial differential equations. Seeking for exact solution of some engineering applications, such as fluid flow problems, is still a challenging task to overcome. Based on this, finite element method can be used to model such problems and it is possible to obtain solution near to the exact one. In the present study, FEM is employed to discretize the governing equations for a viscous incompressible fluid flow around a circular cylinder inside a $2 D$ channel. The fluid flow is described by the NavierStokes equations. There are many methods to tackle these equations. However, minding computational speed the choice is for a simple method called Chorin's projection method for discretizing the Navier-Stokes equations. Results are presented for two different meshes and is shown that the elements density have some significant influence in the results. Also, there is an apparent effect on $\mathrm{Cd}$ and $\mathrm{Cl}$ calculation on the cylinder.
\end{abstract}

Keywords: Finite Element Method (FEM), fluid flow, viscous flow, incompressible flow.

\section{INTRODUCTION}

Finite element method as a numerical technique is widely used in problems that require solution of system of partial differential equations. The core of this method treat a complex region defining a continuum, as a discretized region in simple geometric shapes called finite elements. The governing equations, material properties and auxiliaries' relations are evaluated over these elements and written in terms of unknown values at element vertexes. An assembly process in a correct way is done considering the loading and constraints which results in a set of equations. In order to obtain a solution, the set of these equations are solved yielding the approximate behaviour of the continuum region. Cook [1] and Thompson [2] discuss the concepts of FEM for solution of partial differential equations present in several important areas of engineering as such as, Fluid Mechanics, Solid Mechanic and Electromagnetic Field. Details about fluid mechanics and heat transfer problems and their solution can be found in [3]. 


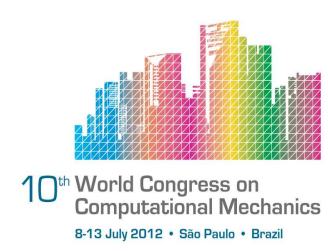

In this paper, a two-dimensional channel with a circular cylinder submitted to a viscous incompressible unsteady flow is solved using the finite element method through the Chorin projection method [4][5]. The 2D channel domain is discretized to a large number of triangular elements in order to assure the accuracy of the solution. The influence of the number of elements inside the domain on the accuracy of the numerical solution is also investigated. Numerical experiments are done by studying different meshes sizes. From these experiments are observed very important results, such as the accuracy of the drag coefficient as well as the lift coefficient on the cylinder geometry.

In the present problem, a fluid flows through a domain represented by a $2 \mathrm{D}$ channel with a circular cylinder. The channel has the following dimensions: $0.41 \mathrm{~m}$ high and $0.22 \mathrm{~m}$ long. The cylinder center is located at $(0.2 \mathrm{~m}, 0.2 \mathrm{~m})$ from the left hand side and has $0.1 \mathrm{~m}$ of diameter. The fluid flow enters from the left with a uniform velocity $u$, $u(0, y)=u(2.2, y)=0.41^{-2}\left(4 u_{\max } y(0.41-y), 0\right), 0 \leq y \leq 0.41$. It flows around a circular cylinder and the outlet is the right side of the domain. The upper and the bottom side are considered as solid walls. No-slip boundary conditions are imposed at these boundaries. As the fluid flows through the domain, the velocity of the fluid changes abruptly due to the presence of the circular cylinder. So, the main goal is capture this flow as time progress. Using the mean value of the inflow velocity $u_{\max }=0.3 \mathrm{~ms}^{-1}$ and the diameter of the cylinder $L=0.1 \mathrm{~m}$ as characteristic quantities of the flow, its Reynolds number is $R e=20$. The density of the fluid is considered as being $\rho=1.0 \mathrm{kgm}^{-3}$. As discussed by Schäfer and Turek [6], due to the non-convex domain $\Omega$, it is unlikely that $(u, p)$ are regular enough such that standard error estimates for higher-order finite element discretizations hold.

\section{GOVERNING EQUATION OF TWO DIMENSIONAL CHANNEL FLOW}

The governing Navier-Stokes equations that govern the two-dimensional channel problem, in general, can be obtained from the following relations

$$
\begin{array}{lcc}
\dot{u}+(u . \nabla) u+\nabla p-v \Delta u=f, & \text { in } \Omega \times I & (a) \\
\nabla . u=0, & \text { in } \Omega \times I & (b) \\
u=g, & \text { in } \Omega \times I & (c) \\
v n . \nabla u-p n=0, & \text { in } \Omega \times I & (d) \\
u=u_{0}, & \text { in } \Omega \times I & (e)
\end{array}
$$

Where $v$ is the viscosity, $v=10^{-3} \mathrm{~ms}^{-1}, \Omega$ is the channel. $u$ and $p$ are the velocity and pressure respectively, and $f$ a given body force. It is assumed that boundary $\partial \Omega$ of the channel domain $\Omega$ is divided into two parts $\Gamma D$ and $\Gamma N$ associated with the no-slip and the do-nothing boundary conditions eq(1.c) and eq(1.d). $g$ is a given function where describe the velocity on $\Gamma D$. Generally, $\Omega$ is a channel and $\Gamma D$ denotes either the rigid walls of the channel, with $g=0$, or the inflow region, with $g$ the inflow velocity profile, while $\Gamma N$ denotes the outlet with the 
boundary condition $v n \cdot \nabla u-p n=0$. The velocity at time $t=0$ is given by the initial condition $u_{0}$ and $I=(0 ; T]$ is the time interval with final time $T$.

\subsection{NUMERICAL SCHEME BASED IN CHORIN'S PROJECTION METHOD}

Following the idea described by Chorin in [4][5] the momentum equation (1.a) is discretized and is called operator splitting in time using the approximation given by forward Euler method. Applying this, the time stepping scheme is obtained as follow

$$
\frac{u_{i, j}^{n+1}-u_{i, j}^{n}}{\Delta t}+\left(u_{i, j}^{n} \cdot \nabla\right) u_{i, j}^{n}+\nabla p_{i, j}^{n}-v \Delta u_{i, j}^{n}=f_{i, j}^{n}
$$

Where $\Delta t$ is the time step. $n$ is the discrete time iterative level. Adding and subtracting an intermediary tentative velocity $u^{*}$ in the discrete time derivative, it is possible write

$$
\frac{u_{i, j}^{n+1}-u_{i, j}^{*}+u_{i, j}^{*}-u_{i, j}^{n}}{\Delta t}+\left(u_{i, j}^{n} . \nabla\right) u_{i, j}^{n}+\nabla p_{i, j}^{n}-v \Delta u_{i, j}^{n}=f_{i, j}^{n}
$$

This expression is satisfied only if

$$
\frac{u_{i, j}^{*}-u_{i, j}^{n}}{\Delta t}=-\left(u_{i, j}^{n} \cdot \nabla\right) u_{i, j}^{n}+v \Delta u_{i, j}^{n}+f_{i, j}^{n}
$$

and also

$$
\frac{u_{i, j}^{n+1}-u_{i, j}^{*}}{\Delta t}=-\nabla p_{i, j}^{n}
$$

holding simultaneously.

The physical reason for the splitting is that with the decoupling of the diffusion and convection of the velocity, the pressure act to enforce the incompressibility constraint. So, considering that $u_{i, j}^{n+1}$, is possible calculate $u_{i, j}^{*}$ from eq(4) separately without having to worry about the pressure. In order to determine the pressure one takes divergence of eq(5). Once done this it is possible write

$$
\nabla \cdot \frac{\left(u_{i, j}^{n+1}-u_{i, j}^{*}\right)}{\Delta t}=-\nabla \cdot\left(\nabla p_{i, j}^{n}\right)
$$

Done this and since one seek for $\nabla \cdot u_{i, j}^{n+1}=0$, the eq(6.0) gives

$$
-\nabla \cdot \frac{u_{i, j}^{*}}{\Delta t}=-\Delta p_{i, j}^{n}
$$

From this the pressure $p_{i, j}^{n+1}$ can be determined from a Poisson type equation. In general eq(7) is frequently referred to as the Pressure Poisson Equation (PPE). Once is given $u_{i, j}^{*}$ it is possible to solve eq(7) in order to get a pressure $p_{i, j}^{n}$ which makes the next velocity $u_{i, j}^{n+1}$ divergence free. Since $p_{i, j}^{n}$ is computed from the tentative velocity $u_{i, j}^{*}$, it is not the actual 


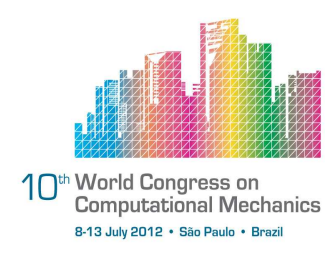

pressure $p$, but at best a first order approximation in time. The actual computation of $u_{i, j}^{n+1}$ is done by making usage of eq(7), but now in the explicit form

$$
u_{i, j}^{n+1}=u_{i, j}^{*}-\Delta t \Delta p_{i, j}^{n}
$$

Thus, with this last calculation the Chorin projection method is established. One simple way to enforce boundary conditions for $u_{i, j}^{*}$ and $p_{i, j}^{n}$ is to set the Dirichlet, or no-slip, velocity boundary conditions given by eq(1.d) on $u_{i, j}^{*}$, and a von Neumann boundary condition $n \cdot \Delta p_{i, j}^{n}=0$ on the pressure. At the outflow, the do-nothing boundary condition given by eq(1.e) is imposed term by term by enforcing $n \cdot \nabla u_{i, j}^{n}=0$ and $p_{i, j}^{n}=0$. This generally means that $u_{i, j}^{n+1}$ will not satisfy the velocity boundary conditions in a precisely sense. The zero Neumann boundary condition for the pressure, which is in some way unphysical and leads to a poor quality of both $p_{i, j}^{n}$ and $u_{i, j}^{n+1}$ near the boundary, is overlooked by the simplicity of implementation of the projection method and has made it very popular and hard to be replaced [7]. These boundary conditions for the pressure have been a great challenge with a long history see e.g. [8] and [9].

\subsection{Fully Discrete Method Appling Finite Elements}

In order to write a fully discrete method it is need to adopt a discretization method, so the finite elements is applied to the Chorin Projection method. To establish a variational formulation of the Navier-Stokes equations we need to introduce fwo function spaces $V$ and $Q$ for the velocity $u$ and pressure $p$, respectively: $V_{g}=\left\{v \in\left[H^{1}(\Omega)\right]^{d}:\left.v\right|_{\partial \Omega}=g\right\}$ and $Q=\left\{q \in L^{2}(\Omega):(q, 1)=0\right\}$, the pressure space $Q$ is the subset of $L^{2}$ functions, which have zero mean. $H^{1}=H^{1}(\Omega)$ is the Hilbert space.

Let further, to approximate the velocity and pressure, let $V_{h}$ and $Q_{h}$ be two spaces of piecewise polynomials on $M e$ (mesh) that approximates $V$ and $Q$ in some sense to be made precise. Consider $V_{h}$ as the usual space of piecewise linears with the hat function basis $\left\{\varphi_{i}\right\}_{i=1}^{n p}$ on a mesh $M e$ of $\Omega$. Also consider $\left\{\mathrm{X}_{i}\right\}_{i=1}^{m p}$ be a set of scalar basis functions for $Q_{h}$. An appreciated goodness with the operator splitting is that it allows one to use equal order polynomial spaces for both the velocity and pressure. This suitably circumvents the cumbersome inf-sup condition as discussed by Tobiska and Verfürth [10]. Even though with this holding, spurious pressure modes may still occur if the time step $\Delta t$ is much smaller than the mesh size $h$ used to discretize $\Omega$. Based on the fact which is adopted the same space for $u$ and $p$, this brings great simplicity when implementing the following relations

$$
\begin{aligned}
& u_{i, j}^{n} \approx \sum_{J=1}^{n p}\left(\xi_{u_{i, j}}^{n}\right)_{J} \varphi_{J} \\
& v_{i, j}^{n} \approx \sum_{J=1}^{n p}\left(\xi_{v_{i, j}^{n}}^{n}\right)_{J} \varphi_{J} \\
& p_{i, j}^{n} \approx \sum_{J=1}^{n p}\left(\tilde{\omega}^{n}\right)_{J} \varphi_{J}
\end{aligned}
$$




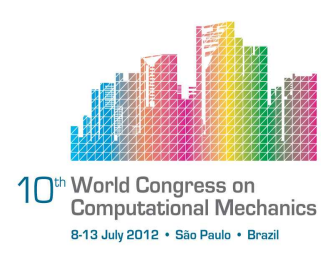

It is imposed the same representation for $u^{*}$.

$* n$

Observing the eq(4) it is possible to see that it decouples into one equation for $u$ and one for $v$. Proceeding with finite element discretization and making usage of matrix notation these equations can be written as

$$
\left\{\begin{array}{l}
M \xi_{u_{i, j}}^{*}=M \xi_{u_{i, j}}^{n}-\Delta t\left(C^{n}+v A\right) \xi_{u_{i, j}}^{n}+b_{u} \\
M \xi_{v_{i, j}}^{*}=M \xi_{v_{i, j}}^{n}-\Delta t\left(C^{n}+v A\right) \xi_{v_{i, j}}^{n}+b_{v}
\end{array}\right.
$$

Where $M$ is the so called mass matrix, $A$ the stiffness matrix, and $C^{n}=C\left(u^{n}\right)$ is the convection matrix with convection field $u^{n}$. Note that $C^{n}$, depends on the current velocity component and must be reassembled at each time step. The load vectors $b$, contain contributions of a previously defined body force $f$. The eq(5) well known as PPE is a standard Poisson equation and rewriting in matrix form yields

$$
A \tilde{\omega}^{n}=-\left(B_{u} \xi_{u}^{*}+B_{v} \xi_{v}^{*}\right) / \Delta t
$$

Where $A$ again is the stiffness matrix. $B$ are convection matrices with corresponding convection fields $[1 ; 0]$ for $u$ and $[0 ; 1]$ for $v$, the two velocity components. It is needed adjust this equation for boundary conditions $\left(\tilde{\omega}=0\right.$ on $\left.\Gamma^{n}\right)$ to give a unique solution. Using the last relation is possible rewrite eq(8.0) in discrete form as

$$
\left\{\begin{array}{l}
M \xi_{u_{i, j}}^{n+1}=M \xi_{u_{i, j}}^{*}-\Delta t B_{u_{i, j}} \tilde{\omega}^{n} \\
M \xi_{v_{i, j}}^{n+1}=M \xi_{v_{i, j}}^{*}-\Delta t B_{v_{i, j}} \tilde{\omega}^{n}
\end{array}\right.
$$

The size of the time step is bounded by the adoption of the first order forward Euler scheme in the time approximation. In order to guarantee numerical stability it is necessary that the time step is of magnitude $h / u$ for convection dominated flow with $v<u h$, and $h^{2} / v$ for diffusion dominated flow with $v \geq u h$.

\section{FLOW ANALYSIS}

Some numerical experiments are carried out using two different unstructured triangular meshes. CASE \#1: number of nodes $=1338$, number of triangles $=2445$, nonuniform triangles. CASE \#2: number of nodes $=10459$, number of triangles $=20155$, uniform triangles. These unstructured triangular meshes were obtained by making use of distmesh $2 d$ by Persson and Strang [11]. The uniform mesh helps the matrixes of finite element to be wellconditioned. In another words, this means that the triangles should not present internal high skew angles. In order to evaluate the accuracy of the present method the usual benchmark parameters are the drag coefficient $c d$ at the cylinder, the lift coefficient $c l$ and are given by the following relations as stated by Schäfer and Turek [6], John and Matthies [12] and an approximation first published for the unsteady Navier-Stokes equations in John [13], which is given as follow 


$$
\begin{aligned}
& c d(t)=\frac{2}{\rho U^{2} L} \int_{S}\left(\rho \nu \nabla u_{t s} n_{y}-p(t) n_{x}\right) d S \\
& c l(t)=-\frac{2}{\rho U^{2} L} \int_{S}\left(\rho \nu \nabla u_{t s} n_{x}+p(t) n_{y}\right) d S
\end{aligned}
$$

Where, $n=\left(n_{x}, n_{y}\right)^{T}$ is the normal vector on the cylinder $\mathrm{S}$ directed into $\Omega$, $t s=\left(n_{y},-n_{x}\right)^{T}$ the tangential vector and $u_{t s}$ the tangential velocity on S. These two integrals have to be computed only in one layer of mesh cells around the cylinder $\mathrm{S}$.

For CASE\#1 is shown whole mesh domain and the circular cylinder where the yellow color highlight it. See figure 1 bellow

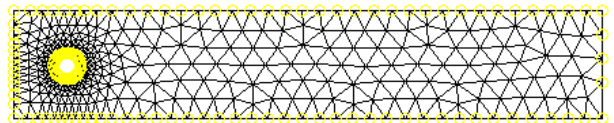

Figure 1. Mesh for CASE\#1

In the figure 2 is shown the velocity flow field.

FEM- 2D Channel: Velocity

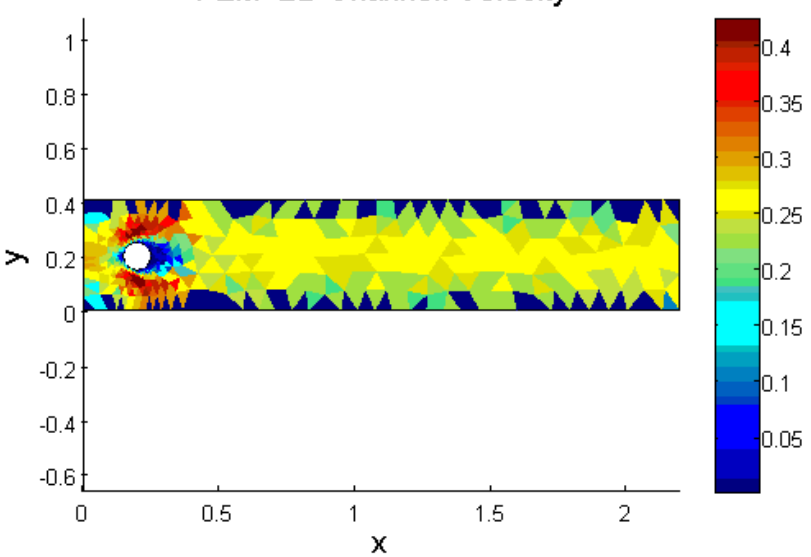

Figure 2. Velocity flow field 
The $c p$ on the circular cylinder is shown in the figure 3 .

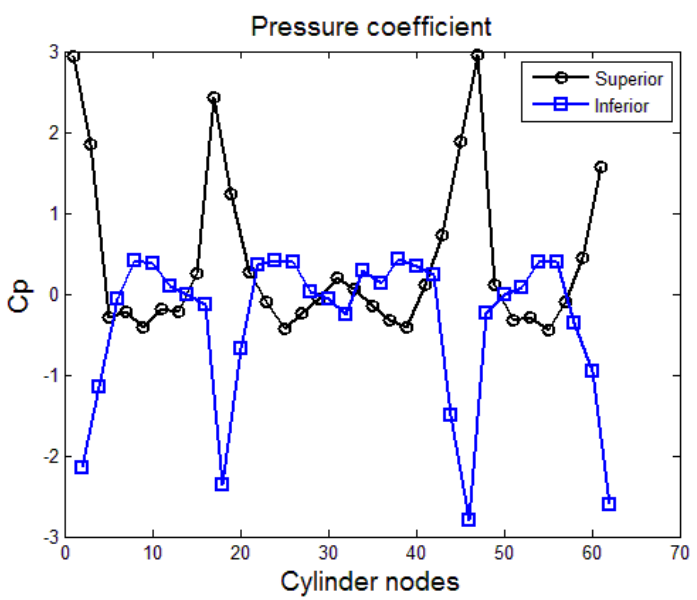

Figure 3. Pressure coefficient on the upper and lower cylinder surface

The drag and lift coefficient after 415 time steps converged to a steady state solution as shown in figure 4 and 5 as follow.

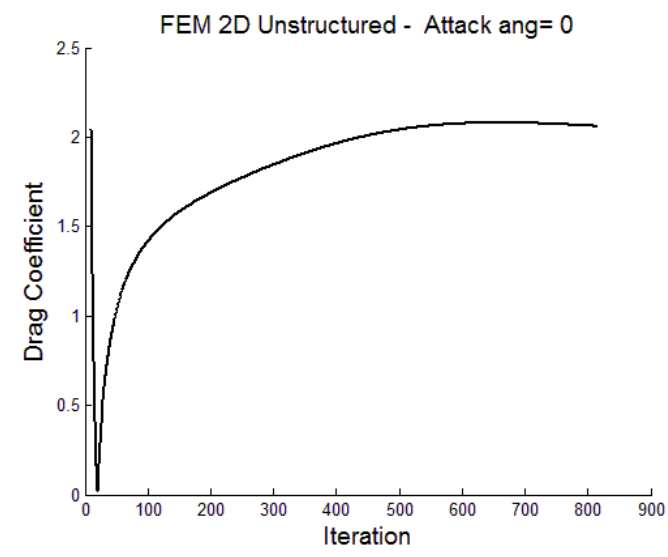

Figure 4. Drag coefficient convergence for CASE\#1 


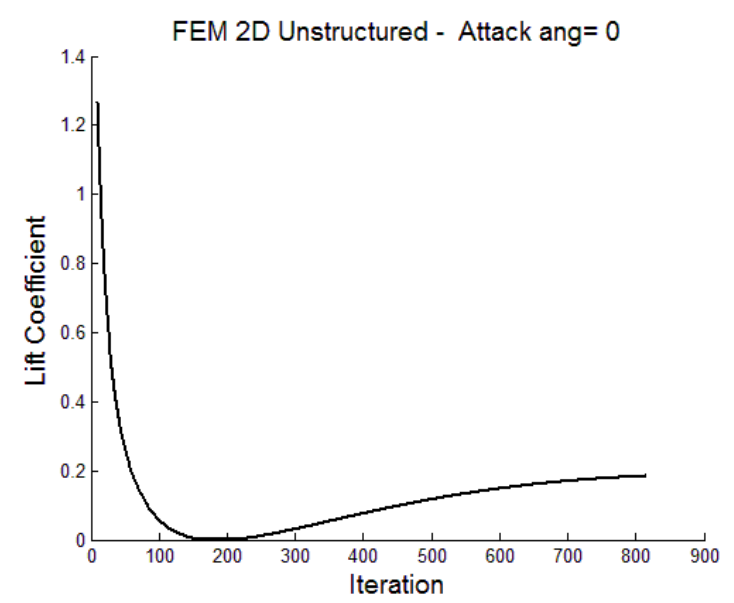

Figure 5. Lift coefficient convergence for CASE\#1

For CASE\#2 the mesh is highly dense as compared as with CASE\#1. See figure 6, as follow

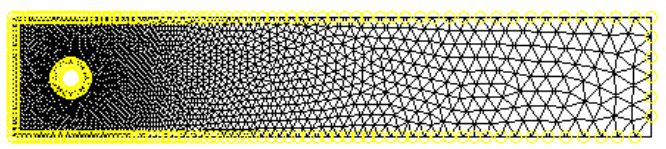

Figure 6. Mesh for CASE\#2 highly dense close the circular cylinder

As can be seen the triangles are more uniform although the mesh doesn't appear to be symmetrical. The drag and lift coefficient after 415 time steps converged to a steady state solution as shown in figure 7 and 8 as follow. 
$\mathrm{O}^{\text {th }}$ World Congress on Computational Mechanics 8-13 July 2012 - São Paulo • Brazil

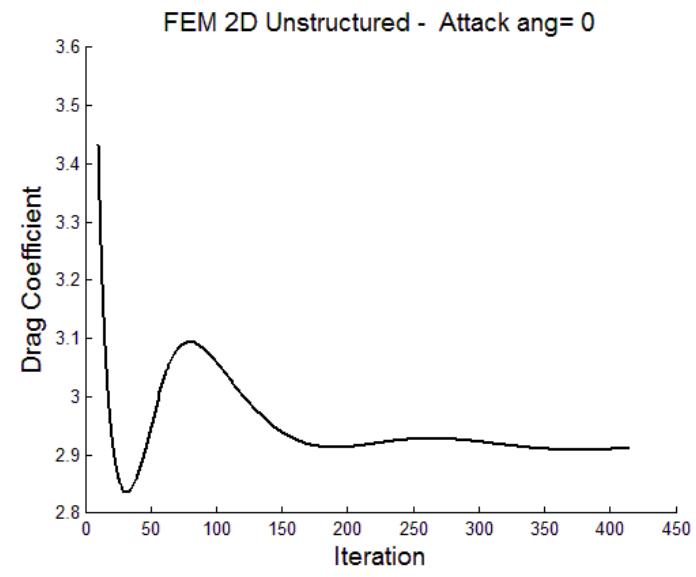

Figure 7. Drag coefficient convergence for CASE\#2

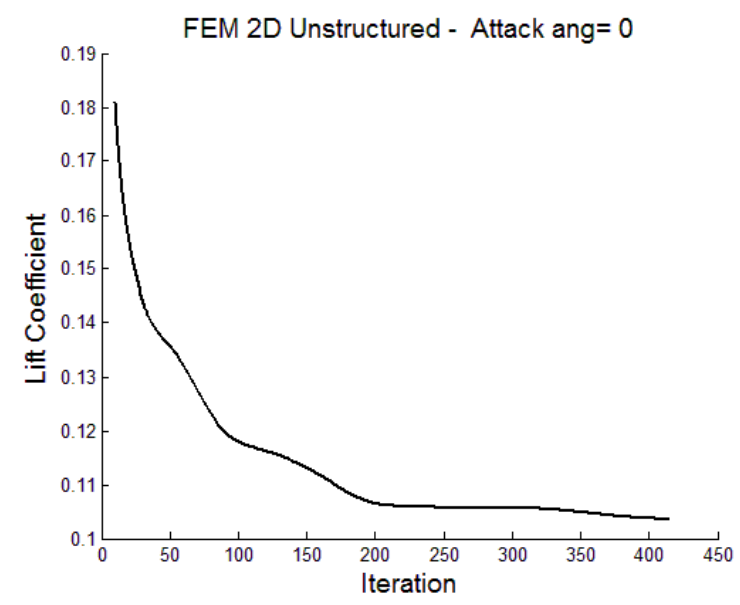

Figure 8. Lift coefficient convergence for CASE\#2 


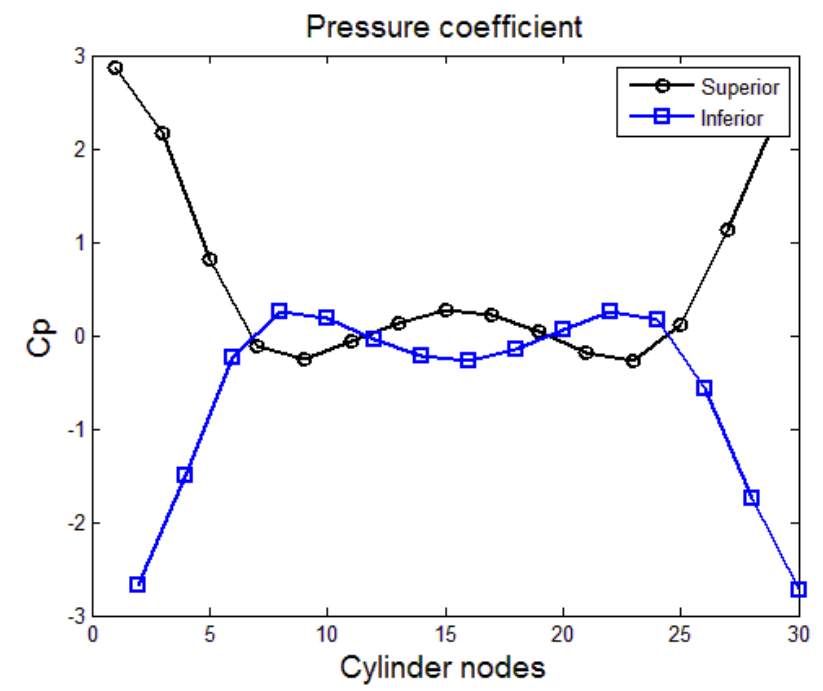

Figure 9. Pressure coefficient on the upper and lower cylinder surface

As there are for this case, more nodes around the cylinder, the $c p$ is more smooth.

The present results as compared with several authors (see table 1 below) show a reasonable accordance. As discussed by Frochte and Heinrichs [14] the most difficult comparison with the benchmark value for several different algorithms is the lift coefficient. The drag coefficient and the pressure are easier to compute presenting closer results. In the present work all cases were simulated with time step of $\Delta t=0.001$ for CASE\#1 and $\Delta t=0.01$ for CASE\#2. It is small enough to take $254,14 \mathrm{~s}$ and $554,13 \mathrm{~s}$ respectively in a dual core simple cpu computer.

Tabela 1. Comparison of drag coefficient $c d$

\begin{tabular}{|l|l|l|l|l|l|l|}
\hline Re & Present Work & $\begin{array}{l}\text { Sucker and } \\
\text { Brauer [15] }\end{array}$ & $\begin{array}{l}\text { Park et al. } \\
{[16]}\end{array}$ & $\begin{array}{l}\text { Ye et al. } \\
{[17]}\end{array}$ & $\begin{array}{l}\text { Tritton } \\
{[18]}\end{array}$ & $\begin{array}{l}\text { Linnick } \\
\text { et al. } \text { [19] }\end{array}$ \\
\hline 20 & $\begin{array}{l}\text { CASE \# 1: } 2.06 \\
\text { CASE \#2: } 2.91\end{array}$ & 2.08 & 2.01 & 2.03 & 2.02 & 2.06 \\
\hline
\end{tabular}

\section{Acknowledgements}

I gratefully acknowledge Federal Fluminese University and Pensa Rio Project for helpful financial support during this work. 


\section{CONCLUSIONS}

In this paper, a channel with a circular cylinder, two-dimensional viscous incompressible unsteady flow problem is analysed using the finite element method by means Chorin's projection method. The fluid flow is expressed by partial differential equations, the Navier-Stokes with appropriate boundary conditions. The flow field of that fluid is then used to solve the Poisson pressure equation. The $2 \mathrm{D}$ domain of the problem is discretized to a large number of triangular-node elements to assure the accuracy of the solution. The influence of the regularity and number of nodes inside the domain on accuracy of the drag and lift coefficient is also investigated. Analyses are done by studying both drag coefficients for two different meshes. Results showed that drag coefficients are influenced considerably by changing the mesh.

\section{REFERENCES}

[1] Cook, R. D., Concepts and Applications of Finite Element Analysis, 2nd ed., John Wiley \& Sons, New York, 1981.

[2] Thompson, Erik G., Introduction to the Finite Element Method: Theory Programming and Applications, John Wiley \& Sons Inc, 2004.

[3] Reddy, J. N., An Introduction to the Finite Element Method, 2nd Ed., McGraw- Hill, New York, 1993.

[4] Chorin, A. J., "A numerical method for solving incompressible viscous flow problems", J. Computational Physics, v. 2, pp. 12, 1967.

[5] Chorin, A. J., "Numerical Solution of the Navier-Stokes Equations", Math. Comp. 22, pp. 745-762, 1968.

[6] Schäfer M., Turek S., "The benchmark problem flow around a cylinder". In Flow Simulation with High-Performance Computers II, Hirschel EH (ed.). Notes on Numerical Fluid Mechanics, vol. 52. Vieweg: Braunschweig. pp. 547-566, 1996.

[7] Quarteroni, A., Saleri, F., Veneziani, A., "Factorization methods for the numerical approximation of Navier-Stokes equations". Comput. Methods Appl. Mech. Engrg. 188, p. 205-526, 2000.

[8] Yanenko, N., N., “The method of fractional steps". Springer-Verlag. Berlin (1971) . Russian ed.: Nauka, Novosibirsk, 1967.

[9] Marchuk, G., I., "Splitting and alternating direction methods. Ciarlet, P. G. (ed.) et al. Handbook of numerical analysis. Volume I: Finite difference methods (Part 1), solution of equations in Rn (Part 1). Amsterdam: North-Holland. pp. 197-462, 1990. 
[10] Tobiska, L., Verfürth R., "Analysis of a streamline diffusion finite element method for the Stokes and Navier-Stokes equations”. SIAM J. Numer. Anal., 1996.

[11] Persson, P.-O., Strang, G., "A Simple Mesh Generator in MATLAB”. SIAM Review, Volume 46 (2), pp. 329-345. June 2004.

[12] John, V., Matthies, G., "Higher order Finite Element discretizations in a benchmark problem for the 3D Navier Stokes equations". Internation Journal for Numerical Methods in Fluid Mechanics, 40, pp.775-798. 2002.

[13] John, V., "Reference values for drag and lift of a two-dimensional time-dependent flow around a cylinder". International Journal for Numerical Methods in Fluids. Int. J. Numer. Meth. Fluids. 44, pp. 777-788. 2004.

[14] Frochte, J., Heinrichs, W., "Journal of Computational and Applied Mathematics", J. COMPUT. APPL. MATH., vol. 228, no. 1, pp. 373-390, 2009.

[15] Sucker, D., Brauer, H., "Fluiddynamik bei der angestromten Zilindern", Wärme Stoffubertragung 8, 149. 1975.

[16] Park,J., Kwon, K., Choi, H., "Numerical solutions of flow past a circular cylinder at Reynolds number up to 160”, KSME Int. J. 12. 1200. 1998.

[17] Ye, T., Mittal, R., Udaykumar, H. S., Shyy, W., "An accurate Cartesian grid method for viscous incompressible flows with complex boundaries”, J. Comp. Phys. 156, 209. 1999.

[18] Triton, D.J., "Experiments on the flow past a circular cylinder at low Reynolds number", J. Fluid Mech. 6, 547. 1959.

[19] Linnick, M. M., Fasel, H. F.,"A high-order immersed interface method for simulating unsteady incompressible flows on irregular domains,” J. Comput. Phys. 204, 157. 2005. 